USC-94-16

SPhT-94/107

hep-th/9409176

\title{
Solving 1d plasmas and 2d boundary problems using Jack polynomials and functional relations
}

\author{
P. Fendley ${ }^{1}$, F. Lesage ${ }^{2}$ and H. Saleur ${ }^{1 *}$ \\ ${ }^{1}$ Department of Physics, University of Southern California \\ Los Angeles CA 90089-0484 \\ 2 Service de Physique Théorique de Saclay \\ F-91191 Gif sur Yvette, France
}

\begin{abstract}
The general one-dimensional "log-sine" gas is defined by restricting the positive and negative charges of a two-dimensional Coulomb gas to live on a circle. Depending on charge constraints, this problem is equivalent to different boundary field theories.

We study the electrically neutral case, which is equivalent to a two-dimensional free boson with an impurity cosine potential. We use two different methods: a perturbative one based on Jack symmetric functions, and a non-perturbative one based on the thermodynamic Bethe ansatz and functional relations. The first method allows us to compute explicitly all coefficients in the virial expansion of the free energy and the experimentallymeasurable conductance. Some results for correlation functions are also presented. The second method provides in particular a surprising fluctuation-dissipation relation between the conductance and the free energy.
\end{abstract}

* Packard Fellow

$9 / 94$ 


\section{Introduction}

The general 2D classical Coulomb gas with charges restricted to live on a circle is a recurrent problem in several areas of theoretical physics. These include random matrix theory [1], impurity problems (like the Kondo effect [2], resonant tunneling in quantum wires [3] and between quantum Hall edge states [4.5]), and dissipative quantum mechanics [6, 17,8,9, 10]. In this $1 \mathrm{~d}$ "log-sine" gas, the charges interact with a long-range interaction proportional to the log of the sine of the separation.

Two particular cases of this Coulomb gas have been solved analytically. The gas with only one type of charge (the Dyson gas) is related to eigenvalue statistics for circular ensembles [1], and can be addressed by elementary methods. When there are two types of charges required to alternate in space [2], the gas is related to the Kondo problem [2], and therefore can be addressed indirectly by the Bethe ansatz solution of the latter [11].

We present in this paper two methods to address the general model with two types of charges. The first method is direct, and uses the recently-developed technical tool of Jack symmetric functions [12,13, 14, 15]. These have been used extensively in recent works on Green functions for the Calogero-Sutherland model [16, 17, 18, 19, 20, 21]. The second method is indirect and uses the solution of the boundary sine-Gordon theory [22] via exact $S$ matrices and the thermodynamic Bethe ansatz (TBA) [23,24].

Technically, the combination of the two methods allows us to compute a rather large number of quantities, including dynamical properties. When they overlap, they can be compared, leading to interesting relations between two different active areas of mathematical physics: $1 / r^{2}$ models and the TBA. Physically, our solution can be applied to a range of interesting problems, including the experimentally-measurable resonant tunneling between quantum Hall edge states (which was derived using the TBA in [5]), and the case of dissipative quantum mechanics which will be discussed elsewhere.

We start by describing this model as a 1+1-dimensional field theory with an impurity. Consider a Gaussian model on an infinite cylinder with action

$$
A=-\frac{1}{2} \int_{-\infty}^{\infty} \int_{0}^{R} d \sigma d \tau\left[\left(\partial_{\tau} \phi\right)^{2}+\left(\partial_{\sigma} \phi\right)^{2}\right]+2 g \int_{0}^{R} d \tau \cos [\beta \phi(\sigma=0, \tau)] .
$$

With periodic boundary conditions in the $\tau$-direction, this is equivalent to a onedimensional quantum problem at non-zero temperature $1 / R$, with an impurity at $\sigma=0$. The impurity coupling $g$ has a dimension, so this problem is not conformally invariant and the interaction induces a flow from the free boson on an infinite cylinder in the UV $(g=0)$ 
to two decoupled free bosons on two half-cylinders with Dirichlet boundary conditions at their boundary in the IR ( $g$ large). A convenient quantity describing this flow is the "g-factor" discussed in [25], whose logarithm is equal to the impurity entropy (the contribution to the entropy which is independent of the length of the cylinder). This "g-factor" (which we prefer to denote $\omega$ here) is $\omega=1$ in the UV and $\omega=t^{-1 / 2}$ in the IR, where we define

$$
t \equiv \frac{4 \pi}{\beta^{2}}
$$

We can study (1.1) by naive perturbation theory, which exhibits the relation with a Coulomb gas. Defining as usual the partition function as $Z=\int[d \phi] e^{A}$ we introduce

$$
\mathcal{Z} \equiv \frac{Z(g)}{Z(g=0)} .
$$

Using the free field propagator on an infinite cylinder

$$
\left\langle\phi(\tau) \phi\left(\tau^{\prime}\right)\right\rangle=-\frac{1}{2 \pi} \ln \left|\frac{\kappa R}{\pi} \sin \frac{\pi}{R}\left(\tau-\tau^{\prime}\right)\right|,
$$

(where $\kappa$ is a renormalization constant) we obtain by the standard perturbation expansion in powers of $g$

$$
\begin{aligned}
\mathcal{Z} & =\sum_{n=0}^{\infty} \frac{g^{2 n}}{(n !)^{2}} \int_{0}^{R} d \tau_{1} \ldots d \tau_{n} d \tau_{1}^{\prime} \ldots d \tau_{n}^{\prime} \\
\times & \frac{\prod_{i<j}\left|\frac{\kappa R}{\pi} \sin \frac{\pi\left(\tau_{i}-\tau_{j}\right)}{R}\right|^{\frac{\beta^{2}}{2 \pi}} \prod_{k<l}\left|\frac{\kappa R}{\pi} \sin \frac{\pi\left(\tau_{k}^{\prime}-\tau_{l}^{\prime}\right)}{R}\right|^{\frac{\beta^{2}}{2 \pi}}}{\prod_{i, k}\left|\frac{\kappa R}{\pi} \sin \frac{\pi\left(\tau_{i}-\tau_{k}^{\prime}\right)}{R}\right|^{\frac{\beta^{2}}{2 \pi}}} .
\end{aligned}
$$

The sum (1.2) is the grand canonical partition function for a classical two-dimensional Coulomb gas with two species of particles (with positive and negative charges) that lie on a circle of radius $R$, and which is electrically neutral. The parameter $g$ is the fugacity of charges, while $\beta$ controls the combination charge ${ }^{2} \times$ inverse temperature. In sect. 2 we will show how these integrals can be explicitly evaluated by expanding the integrands in terms of Jack polynomials.

We can also reformulate this as a boundary problem (i.e. on the half-cylinder) following [26]. We introduce two new fields

$$
\begin{aligned}
& \phi_{e}(\sigma, \tau)=\frac{1}{\sqrt{2}}[\phi(\sigma, \tau)+\phi(-\sigma, \tau)] \\
& \phi_{o}(\sigma, \tau)=\frac{1}{\sqrt{2}}[\phi(\sigma, \tau)-\phi(-\sigma, \tau)],
\end{aligned}
$$


so the action reads now

$$
\begin{gathered}
A=-\frac{1}{2} \int_{0}^{\infty} \int_{0}^{R} d \sigma d \tau\left[\left(\partial_{\tau} \phi_{e}\right)^{2}+\left(\partial_{\sigma} \phi_{e}\right)^{2}+\left(\partial_{\tau} \phi_{o}\right)^{2}+\left(\partial_{\sigma} \phi_{o}\right)^{2}\right] \\
+2 g \int_{0}^{R} d \tau \cos \left[\frac{\beta}{\sqrt{2}} \phi_{e}(\sigma=0, \tau)\right] .
\end{gathered}
$$

One can of course obtain the perturbative expansion (1.2) from (1.3) by using the propagator on the half-cylinder. In the UV both fields have Neumann boundary conditions and $\omega=\left(\frac{2}{t}\right)^{1 / 2}$ while in the IR the odd field has still Neumann boundary conditions while the even has Dirichlet boundary conditions and $\omega=\frac{1}{\sqrt{2}}$. Notice that in the reformulation the absolute values of $\omega$ have changed; this is presumably due to Jacobian terms in the functional integral when redefining the new fields. However, the ratio $\frac{\omega_{U V}}{\omega_{I R}}=\frac{\omega_{D}}{\omega_{N}}=t^{-1 / 2}$ remains a constant.

Using this reformulation, the free energy $\mathcal{F}=-T \ln \mathcal{Z}$ can be obtained nonperturbatively using the thermodynamic Bethe ansatz [23,24]. The corresponding analysis appears in 22 for $t$ integer. We will discuss this in sect. 4, where we will also derive a set of functional equations which $\mathcal{Z}$ satisfies. The results of TBA depend on a dimensionless variable $T / T_{B}$, where

$$
g=\kappa^{\prime} T_{B}^{\frac{t-1}{t}}
$$

and $\kappa^{\prime}$ is an unknown renormalization constant. The non-perturbative free energy also contains a non-analytic term $\overline{\mathcal{F}}$ independent of $T$, which the detailed analysis gives as

$$
\overline{\mathcal{F}}=\frac{T_{B}}{2 \cos (\pi / 2(t-1))}
$$

There is also a shift because we have $\mathcal{F}(g=0)=0$, whereas the TBA is defined so that $\mathcal{F}_{T B A}(g \rightarrow \infty) \longrightarrow 0$. Thus

$$
\mathcal{F}_{T B A}=\mathcal{F}-T \ln \sqrt{t}+\overline{\mathcal{F}}
$$

This allows us to obtain the exact behavior of the free energy at large $g$ (large $T_{B}$ ), because the the power series must be precisely balanced by $\overline{\mathcal{F}}$, and thus

$$
\mathcal{Z} \approx \frac{1}{\sqrt{t}} \exp \left(\frac{T_{B}}{2 \cos (\pi / 2(t-1))}\right)
$$

in this limit. We will see in sect. 2 that this behavior also follows from the expansion (1.2). 
This two-dimensional local field theory problem can also be reformulated as a onedimensional non-local field theory on a circle of circumference $R$ by integrating out the "bulk" degrees of freedom. Let us consider for instance the second (boundary) point of view and forget about the odd field which totally decouples. After integration the even action reads

$$
A_{e}^{b d r y}=-\frac{\pi}{2 R^{2}} \int_{0}^{R} \int_{0}^{R} d \tau d \tau^{\prime} \frac{\phi_{e}(\tau) \phi_{e}\left(\tau^{\prime}\right)}{\left[\sin \frac{\pi}{R}\left(\tau-\tau^{\prime}\right)\right]^{2}}+2 g \int_{0}^{R} d \tau \cos \left[\frac{\beta}{\sqrt{2}} \phi_{e}(\tau)\right] .
$$

We therefore have a one-dimensional model with a sine-Gordon-type interaction, where the Gaussian part has a $1 / r^{2}$ interaction. Related models have been considered in [27,28].

\section{Exact solution of the Coulomb gas problem using Jack polynomials}

In this section we derive all the coefficients of the perturbative expansion of the partition function. The result (1.2) can be easily recast after a change of integration variables into

$$
\mathcal{Z}=\sum_{n=0}^{\infty} x^{2 n} Z_{2 n}
$$

where we have set

$$
x=R g\left(\frac{\kappa R}{2 \pi}\right)^{-\frac{1}{t}}
$$

and

$$
\begin{aligned}
Z_{2 n} & \equiv \frac{1}{(n !)^{2}} \int_{0}^{2 \pi} \frac{d u_{1}}{2 \pi} \ldots \frac{d u_{n}}{2 \pi} \frac{d u_{1}^{\prime}}{2 \pi} \ldots \frac{d u_{n}^{\prime}}{2 \pi}\left|\frac{\prod_{i<j} 2 \sin \left(\frac{u_{i}-u_{j}}{2}\right) \prod_{k<l} 2 \sin \left(\frac{u_{k}^{\prime}-u_{l}^{\prime}}{2}\right)}{\prod_{i, k} 2 \sin \left(\frac{u_{i}-u_{k}^{\prime}}{2}\right)}\right|^{2 / t} \\
& =\frac{1}{(n !)^{2}} \oint \prod_{i}\left(\frac{d z_{i}}{2 i \pi z_{i}} \frac{d z_{i}^{\prime}}{2 i \pi z_{i}^{\prime}}\right) \frac{(\Delta(z) \overline{\Delta(z)})^{1 / t}\left(\Delta\left(z^{\prime}\right) \overline{\Delta\left(z^{\prime}\right)}\right)^{1 / t}}{\prod_{i, k}\left[\left(1-z_{i} \overline{z_{k}^{\prime}}\right)\left(1-z_{k}^{\prime} \overline{z_{i}}\right)\right]^{1 / t}}
\end{aligned}
$$

where $\Delta(z)$ is the $n$-variable Vandermonde determinant $\Delta(z)=\prod_{i<j}\left(z_{i}-z_{j}\right)$, and $z_{k}=$ $e^{i u_{k}}$.

To evaluate this integral, we expand the integrand in terms of Jack polynomials $14,12,13$

$$
\prod_{i, j} \frac{1}{\left(1-r_{i} s_{j}\right)^{a}}=\sum_{\lambda} b_{\lambda}(a) P_{\lambda}(r, a) P_{\lambda}(s, a) .
$$

The function $P_{\lambda}(r, a)$ is a symmetric polynomial in the set of variables $\left(r_{1}, r_{2}, \ldots, r_{n}\right)$ which depends on a rational number $a$. The subscript $\lambda$ is a partition of an integer; this is 
conveniently labeled by a Young tableau; e.g. the partition $5=2+2+1$ is labeled by the tableau with two boxes in the first row, two boxes in the second, and one in the third. The polynomials $P_{\lambda}(x, a)$ vanish if the number of parts $l(\lambda)$ of the partition $\lambda$ (i.e. the number of rows of the tableau) is greater than the number $n$ of variables, so the sum runs over all partitions of all integers with $l(\lambda) \leq n$. The Jack polynomials have the useful property that their orthogonality relation involves the Vandermonde determinant:

$$
\oint \prod_{i} \frac{d z_{i}}{2 i \pi z_{i}}(\Delta(z) \overline{\Delta(z)})^{a} P_{\lambda}(z, a) P_{\mu}(\bar{z}, a)=\delta_{\lambda, \mu} N_{\lambda}(a)
$$

Hence the value of the integral follows (here $a=1 / t$ ) :

$$
Z_{2 n}=\frac{1}{(n !)^{2}} \sum_{\substack{\lambda \\ l(\lambda) \leq n}} b_{\lambda}^{2} N_{\lambda}^{2}
$$

The numerical coefficients in the foregoing expression are expressed as a product over the boxes of the Young tableau associated with the partition $\lambda$ [14, 12, 13]. We have

$$
b_{\lambda}^{2} N_{\lambda}^{2}=c_{n}^{2} \prod_{s \in \lambda}\left(\frac{j-1+\frac{1}{t}(n-i+1)}{j+\frac{1}{t}(n-i)}\right)^{2}
$$

where

$$
c_{n}=\frac{\Gamma\left(\frac{1}{t} n+1\right)}{\left[\Gamma\left(1+\frac{1}{t}\right)\right]^{n}}
$$

and $s=(i, j)$ is the box of the tableau at the intersection of the $j^{\text {th }}$ column and $i^{\text {th }}$ line.

One can write this product compactly using gamma functions. Two convenient expressions of $Z_{2 n}$ follow, depending on whether one uses partitions or their conjugates (given by interchanging the rows and columns of the Young tableau). One obtains

$$
Z_{2 n}=\left(\frac{c_{n}}{n !}\right)^{2} \sum_{\substack{\lambda \\ l(\lambda) \leq n}} \prod_{i=1}^{l(\lambda)}\left[\frac{\Gamma\left(\frac{1}{t}(n-i)+1\right) \Gamma\left(\frac{1}{t}(n+1-i)+\lambda_{i}\right)}{\Gamma\left(\frac{1}{t}(n-i+1)\right) \Gamma\left(\frac{1}{t}(n-i)+1+\lambda_{i}\right)}\right]^{2}
$$

and alternatively, using the conjugates,

$$
Z_{2 n}=\left(\frac{c_{n}}{n !}\right)^{2} \sum_{\substack{\lambda \\ l(\lambda) \leq n}} \prod_{i=1}^{\lambda_{1}}\left(\frac{\Gamma(n+1+t(i-1))}{\Gamma(n+t i)}\right)^{2}\left(\frac{\Gamma\left(n-\lambda_{i}^{\prime}+t i\right)}{\Gamma\left(n-\lambda_{i}^{\prime}+1+t(i-1)\right)}\right)^{2}
$$

where $\lambda_{i+1} \leq \lambda_{i}$. 
Consider for instance the case $n=1$ : (2.7) reads then

$$
Z_{2}(t)=\sum_{\lambda_{1}=0}^{\infty}\left(\frac{\Gamma\left(1 / t+\lambda_{1}\right)}{\Gamma(1 / t) \Gamma\left(1+\lambda_{1}\right)}\right)^{2}
$$

The sum converges only for $t>2$, so that the UV dimension of the perturbing operator $x=\frac{\beta^{2}}{4 \pi}<\frac{1}{2}$ [29]. This coincides with the domain where the integrals in (1.2) are UV convergent. (We have no problem with IR divergences because we are on a circle.) The sum in (2.9) can be done explicitly; one can also obtain its value by a direct treatment of the integral avoiding Jack functions. The result is

$$
Z_{2}(t)=\frac{\Gamma(1-2 / t)}{\Gamma^{2}(1-1 / t)}
$$

Let us now study the large- $n$ behavior of the series (1.2). Using the expression with conjugate partitions 2.8 we find

$$
Z_{2 n}=\left(\frac{c_{n}}{n !}\right)^{2} \sum_{\substack{\lambda \\ l(\lambda) \leq n}} \prod_{i=1}^{\lambda_{1}}\left(\frac{\left(n-\lambda_{i}^{\prime}+t i-1\right)\left(n-\lambda_{i}^{\prime}+t i-2\right) \ldots\left(n-\lambda_{i}^{\prime}+t i-t+1\right)}{(n+t i-1)(n+t i-2) \ldots(n+t i-t+1)}\right)^{2} .
$$

Since conjugate partitions are limited by the number $n$ we can approximate the sum in (2.8) for large $n$ as an integral over the variables $\frac{\lambda_{i}^{\prime}}{n} \equiv v_{i}$. Calling the number of boxes in the first line $\lambda_{1} \equiv p$ we get :

$$
Z_{2 n} \simeq\left(\frac{c_{n}}{n !}\right)^{2} \sum_{p=0}^{\infty} N^{p} \int_{0}^{1} d v_{1} \int_{0}^{v_{1}} d v_{2} \ldots \int_{0}^{v_{p-1}} d v_{p} \prod_{i=1}^{p}\left(1-\frac{v_{i}}{\left(1+\frac{t i}{N}\right)}\right)^{2 t-2}
$$

The integrand is minimized by $\left(1-v_{i}\right)^{2 t-2}$ and maximized by 1 . In both cases one can symmetrize over the $v_{i}$ to compute the integral explicitly and one finds :

$$
e^{n /(2 t-1)}\left(\frac{c_{n}}{n !}\right)^{2}<Z_{2 n}<e^{n}\left(\frac{c_{n}}{n !}\right)^{2} .
$$

Therefore, the large- $n$ behavior of the $Z_{2 n}$ is fully controlled by the $\left(c_{n} / n !\right)^{2}$ prefactor and

$$
Z_{2 n} \approx \exp \left[2 n\left(\frac{1}{t}-1\right) \log n+O(n)\right]
$$

An important conclusion is that for $t>2$ the radius of convergence of (2.1) is infinite. Moreover approximating the sum over $n$ by an integral we find

$$
\mathcal{Z} \approx \exp \left(\text { constant } x^{\frac{t}{t-1}}\right)
$$


in agreement with (1.6). This behavior is well expected on physical grounds. Indeed the partition function reads also $\mathcal{Z}=\exp \left(\frac{\mathcal{E}}{T}-\mathcal{S}\right)$ where $T=1 / R$ is the temperature of the equivalent one-dimensional quantum system, and $\mathcal{E}$ and $\mathcal{S}$ are the impurity energy and entropy respectively. In the deep IR the impurity entropy converges to $s \rightarrow \ln \omega$ and the energy, on dimensional grounds, scales as $g^{\frac{t}{t-1}}$. The behavior (2.12) is the analog of the "bulk term" in flows between bulk critical points.

Although expressions (2.7) or (2.8) are in effect a solution of the problem, one can wonder about their practical use. Trying to evaluate the $Z_{2 n}$ numerically, one finds that the series converges very slowly. For example, for $t=3$, evaluating a billion terms gives an accuracy of only about .1\%. Fortunately, results are greatly improved by studying the free energy $\mathcal{F}=-T \ln \mathcal{Z}$, whose expansion we write

$$
\mathcal{F}=T \sum_{n=0}^{\infty} f_{2 n} x^{2 n}
$$

The $f_{2 n}$ are of course given in terms of the $Z_{2 n}$. For example, $f_{2}=-Z_{2}$, and $f_{4}=$ $-Z_{4}+Z_{2}^{2} / 2$. When evaluating the $f_{2 n}$ numerically for $n>1$, we find a much faster convergence. For $t=3$, we have

$$
\begin{array}{ll}
f_{2}=-\Gamma(1 / 3) / \Gamma^{2}(2 / 3) & f_{8}=0.044223558 \\
f_{4}=0.229454064 & f_{10}=-0.022852208 \\
f_{6}=-0.092261103 & f_{12}=0.012329254
\end{array}
$$

The $Z_{2 n}$ can be extracted from these data, and are

$$
\begin{array}{ll}
Z_{2}=\Gamma(1 / 3) / \Gamma^{2}(2 / 3) & Z_{8}=0.0618476490 \\
Z_{4}=0.8378042270 & Z_{10}=0.01021005440 \\
Z_{6}=0.276783312 & Z_{12}=0.00131673987
\end{array}
$$

These coefficients are enough to get a good approximation of the properties all the way to the infrared (very large $x$ ) using Pade approximants. It is then preferable to consider the entropy $\mathcal{S}=\frac{\partial \mathcal{F}}{\partial T}$, which is bounded for $x$ large. Keeping the coefficients through $f_{12}$, one finds for instance that $\mathcal{S}(x=0)-\mathcal{S}(x=\infty) \approx .57$, in good agreement with the exact value $\ln \sqrt{3} \approx .549306 \ldots$ 


\section{Other quantities of interest}

The previous calculation of the partition function is the simplest calculation which can be done using Jack symmetric functions. In this section we present several other calculations, and present a conjecture for the experimentally-measurable conductance.

\subsection{Twisted partition functions}

We have so far considered a periodic field $\phi$ on the cylinder. We could also have winding modes such that

$$
\phi(\sigma, \tau+R)=\phi(\sigma, \tau)+\frac{2 \pi}{\beta} p,
$$

where $p$ is an integer. By splitting the field into classical and quantum parts we obtain an action similar to (1.1) but the interaction term is now

$$
2 g \int_{0}^{R} d \tau \cos \left[\beta \phi(\sigma=0, \tau)+2 \pi \frac{p}{R} \tau\right]
$$

Defining as before $\mathcal{Z}(p) \equiv Z(g, p) / Z(g=0, p)$ we find a perturbative expansion similar to (1.2) with however each term in the sum multiplied by

$$
\exp \left[i 2 \pi \frac{p}{R}\left(\tau_{1}+\ldots+\tau_{n}-\tau_{1}^{\prime} \ldots-\tau_{n}^{\prime}\right)\right] .
$$

After change of variables we have the same expansion as (2.1) but with

$$
Z_{2 n}(p) \equiv \frac{1}{(n !)^{2}} \oint \prod_{i}\left(\frac{d z_{i}}{2 i \pi z_{i}} \frac{d z_{i}^{\prime}}{2 i \pi z_{i}^{\prime}}\right) \frac{(\Delta(z) \overline{\Delta(z)})^{\frac{1}{t}}\left(\Delta\left(z^{\prime}\right) \overline{\Delta\left(z^{\prime}\right)}\right)^{\frac{1}{t}}}{\prod_{i, k}\left[\left(1-z_{i}{\overline{z_{k}}}^{\prime}\right)\left(1-z_{k}^{\prime} \bar{z}_{i}\right)\right]^{\frac{1}{t}}}\left(\frac{z_{1} \ldots z_{n}}{z_{1}^{\prime} \ldots z_{n}^{\prime}}\right)^{p}
$$

so in the Coulomb gas language there is now a magnetic charge located at the center of the circle. (We assume $p$ is positive, otherwise just replace $p$ by $|p|$.) We now use the fact that

$$
\left(z_{1} \ldots z_{n}\right)^{p} P_{\lambda}(z, a)=P_{\lambda+p}(z, a)
$$

where $\lambda+p$ means the partition $\lambda$ where $p$ columns of length $n$ have been added. Therefore

$$
Z_{2 n}(p)=\frac{1}{(n !)^{2}} \sum_{\substack{\lambda \\ l(\lambda) \leq n}} b_{\lambda} b_{\lambda+p} N_{\lambda}^{2}
$$

where we use the relation $N_{\lambda}=N_{\lambda+p}$ that follows immediately from the integral defining the norm $N$. The coefficients $b_{\lambda}$ read

$$
b_{\lambda}=\prod_{s \in \lambda} \frac{\lambda_{i}-j+\frac{1}{t}\left(\lambda_{j}^{\prime}-i+1\right)}{\lambda_{i}-j+1+\frac{1}{t}\left(\lambda_{j}^{\prime}-i\right)}
$$


For instance one has

$$
Z_{2}(z)=\sum_{\lambda_{1}=0}^{\infty} \frac{\Gamma\left(\frac{1}{t}+\lambda_{1}\right) \Gamma\left(\frac{1}{t}+\lambda_{1}+z\right)}{\Gamma^{2}\left(\frac{1}{t}\right) \Gamma\left(1+\lambda_{1}\right) \Gamma\left(1+\lambda_{1}+z\right)}=\frac{\sin \frac{\pi}{t} \Gamma\left(1-\frac{2}{t}\right)}{\sin \pi\left(\frac{1}{t}+z\right) \Gamma\left(1-\frac{1}{t}+z\right) \Gamma\left(1-\frac{1}{t}-z\right)},
$$

for $x$ an arbitrary real number, and when $x=p$ is an integer,

$$
Z_{2}(p)=\frac{(-1)^{p} \Gamma\left(1-\frac{2}{t}\right)}{\Gamma\left(1-\frac{1}{t}+p\right) \Gamma\left(1-\frac{1}{t}-p\right)} .
$$

We can also compute the partition function when electrical neutrality is broken by some amount $Q$ (assumed integer). Defining

$$
\mathcal{Z}_{\mathcal{Q}}=\lim _{\sigma \rightarrow \infty} \sigma^{Q \frac{\beta^{2}}{2 \pi}} \frac{\int[d \phi] e^{i Q \phi(\sigma, 0)} e^{A}}{Z(g=0)}
$$

we have

$$
\mathcal{Z}_{\mathcal{Q}}=\sum_{n=0}^{\infty} x^{2 n+Q} Z_{n, n+Q}
$$

where $Z_{n, n+Q}$ has formally the same expression as (2.2) but there are $n$ variables $z$ and $n+Q$ variables $z^{\prime}$. By the same manipulations we obtain

$$
Z_{n, n+Q}=\frac{1}{n !(n+Q) !} \sum_{\substack{\lambda \\ l(\lambda) \leq n}} b_{\lambda}(n) b_{\lambda}(n+Q) N_{\lambda}(n) N_{\lambda}(n+Q) .
$$

Observe that for $n=0$ we recover the well-known expression [1]

$$
Z_{0, Q}=b_{0}(Q) N_{0}(Q)=c_{Q}
$$

\subsection{Correlation functions and the conductance}

The tool of Jack polynomials should allow the perturbative evaluation of correlation functions. However the calculation requires the knowledge of branching coefficients which are not known yet in general. We will illustrate this with an example. Consider the two-point function of the field $\phi$ itself. By the same perturbative approach we find

$$
\left\langle\phi(\tau) \phi\left(\tau^{\prime}\right)\right\rangle_{g}=\left\langle\phi(\tau) \phi\left(\tau^{\prime}\right)\right\rangle_{0}+\frac{1}{\mathcal{Z}} \frac{1}{4 \pi t} \sum_{p=1}^{\infty} \frac{x^{2 p}}{(p !)^{2}} \sum_{n=1}^{\infty} \frac{\left(\bar{z} z^{\prime}\right)^{n}+\left(z \overline{z^{\prime}}\right)^{n}}{n^{2}} \mathcal{C}_{n p}
$$

where

$$
\mathcal{C}_{n p} \equiv \oint \prod_{i=1}^{p}\left(\frac{d z_{i}}{2 i \pi z_{i}} \frac{d z_{i}^{\prime}}{2 i \pi z_{i}^{\prime}}\right) \frac{(\Delta(z) \overline{\Delta(z)})^{1 / t}\left(\Delta\left(z^{\prime}\right) \overline{\Delta\left(z^{\prime}\right)}\right)^{1 / t}}{\prod_{i, k}\left[\left(1-z_{i} \overline{z k}^{\prime}\right)\left(1-z_{k}^{\prime} \bar{z}_{i}\right)\right]^{1 / t}} R_{n}\left(z_{i}, z_{i}^{\prime}\right) R_{n}\left(\bar{z}_{i}^{\prime}, \bar{z}_{i}\right)
$$




$$
R_{n}\left(x_{i}, y_{i}^{\prime}\right) \equiv \sum_{i}\left(x_{i}^{n}-y_{i}^{n}\right)
$$

The calculation can be easily done for the first term $p=1$ using (3.3). One finds following the same lines as in the previous paragraph

$$
\mathcal{C}_{n 1}=2\left(Z_{2}(n)-Z_{2}(0)\right)
$$

For general $p$, we can still decompose the Coulomb-interaction term between positive and negative charges using Jack polynomials as in (2.3). However, we cannot use the relation (2.4) since we have the extra factors $R_{n}$. We can decompose 21, 15

$$
\sum_{i} z_{i}^{n}=\frac{n}{a} \sum_{|\lambda|=n} \frac{\prod_{(i, j) \neq(1,1)}[(j-1) / a-(i-1)]}{\prod_{(i, j)}\left[\lambda_{j}^{\prime}-i+\left(\lambda_{i}-j+1\right) / a\right]} P_{\lambda}(z, a)
$$

For our problem $a=\frac{\beta^{2}}{4 \pi}=1 / t$ and the only tableaux which contribute are the ones of the form $\lambda_{1} \geq \lambda_{2}, \ldots \geq \lambda_{t}, 1, \ldots, 1$. The Jack polynomials in (3.11) multiply the ones in (2.3). We therefore end up with the problem of determining the coupling coefficients

$$
P_{\lambda} P_{\mu}=\sum_{\nu} g_{\lambda \mu}^{\nu} P_{\nu}
$$

Unfortunately these coefficients are not yet known in general.

The two-point function of the field $\phi$ is especially useful in physical applications: in the $2 \mathrm{~d}$ boundary problem the Kubo formula relates it to the conductance [3], while in dissipative quantum mechanics it is the mobility [8,9]. The conductance at the Matsubara frequency $\omega_{n}=\frac{2 \pi}{R} n$ follows from

$$
G_{n}=\frac{2 \omega_{n}}{t} \int_{0}^{R} d \tau^{\prime}\left\langle\phi(\tau) \phi\left(\tau^{\prime}\right)\right\rangle e^{\frac{2 i \pi}{R} n\left(\tau-\tau^{\prime}\right)}
$$

and the $g^{2}$ term is easily picked up in (3.10). The DC conductance then is obtained by analytically continuing $(3.12)$ to $n=0$, leading to

$$
G=\frac{1}{t}+2 \frac{x^{2}}{t^{2}} \operatorname{Lim}_{n \rightarrow 0} \frac{Z_{2}(n)-Z_{2}(0)}{n}+O\left(x^{4}\right)
$$

Using (3.5) it is easy to perform the limit and one finds finally

$$
G=\frac{1}{t}-\frac{x^{2}}{t^{2}} 2^{1-\frac{2}{t}} \frac{\Gamma\left(\frac{1}{t}\right) \Gamma\left(\frac{1}{2}\right)}{\Gamma\left(\frac{1}{2}+\frac{1}{t}\right)}
$$


in agreement with integral done without the Jack functions [3].

We cannot for the moment compute the two-point function to all orders. However, we have the following conjecture for $G$ to all orders:

$$
G=\frac{1}{t}+\left.\frac{2 x}{t^{2}} \frac{d^{2}}{d p d x} \ln Z(g, p)\right|_{p=0},
$$

so the first few terms read then

$$
G=\frac{1}{t}+\frac{2}{t^{2}}\left(Z_{2}^{\prime} x^{2}+2\left(Z_{4}^{\prime}-Z_{2} Z_{2}^{\prime}\right) x^{4}+3\left(Z_{6}^{\prime}-Z_{2}^{\prime} Z_{4}-Z_{4} Z_{2}^{\prime}+Z_{1}^{\prime} Z_{2}^{2}\right) x^{6}+\ldots\right)
$$

We define the continuation of $Z_{2 n}(p)$ to real values of $p$ by simple substitution in (3.4), so

$$
\begin{aligned}
Z_{2 n}^{\prime} & \left.\equiv \frac{d}{d z} Z_{2 n}(z)\right|_{z=0} \\
& =\frac{1}{(n !)^{2}} \sum_{\substack{\lambda \\
l(\lambda) \leq n}} b_{\lambda}^{2} N_{\lambda}^{2}\left[\sum_{i=1}^{n} \psi\left(\lambda_{i}+\frac{1}{t}(n-i+1)\right)-\psi\left(\lambda_{i}+1+\frac{1}{t}(n-i)\right)\right],
\end{aligned}
$$

where $\psi(z)=\Gamma(z)^{\prime} / \Gamma(z)$. We can investigate these numbers numerically. For $t=3$, (3.15) gives $Z_{2}^{\prime}=-2.64996, Z_{4}^{\prime}=-2.351$, and $Z_{6}^{\prime}=-0.964$. The first agrees with (3.13), and the

others are in very good agreement with the conductance calculated using the TBA in the next section. The conjecture (3.14) appears to be a reasonable form of the Kubo formula [3], but we have not succeeded in deriving it rigorously.

To close this section we would like to remark that, although the theory of Jack symmetric functions generally deals with rational values of $t$, we expect all formulas obtained above to hold for any $t>2$ by naive substitution.

\section{Non-perturbative treatment}

The boundary problem (1.3) is integrable [30]. One can thus find the exact $S$ matrix for the quasiparticles of the problem [30], and then use the thermodynamic Bethe ansatz [23,24] to compute the free energy [22] and the conductance [5]. In this section, we describe these results, and use them to derive a variety of functional equations. These functional relations give non-perturbative equations for the free energy, and allow one to derive simple but non-trivial relations among the coefficients $Z_{2 n}$. Another functional relation relates the conductance to the free energy, thus giving a new fluctuation-dissipation theorem for this 
system. We also find expansion coefficients at $t=3$ numerically from the TBA as another check on our results.

The starting point of the TBA is the quasiparticle description of a two-dimensional integrable field theory. These quasiparticles scatter among themselves and off of the impurity with a known $S$ matrix. At any value of $t$, the quasiparticle spectrum includes the soliton and antisoliton, which we label by + and - respectively. Moreover, at coupling $t$, there are $t-2$ "breather" states in the spectrum. The energy and momentum of these left-moving massless particles are parametrized by rapidity variable $\theta$, so $E=-P=\mu_{r} \exp (-\theta)$, where $\mu_{+}=\mu_{-}=\mu$ and $\mu_{j}=2 \mu \sin (\pi / 2(t-1))$ for the breathers. We define the density of states $n_{r}$ and the density of filled states $\rho_{r}$ for each quasiparticle species $r$. Periodic boundary conditions gives the $n_{r}$ as a functional of the $\rho_{r}$. The free energy can be written in terms of these quantities; demanding it be at a minimum gives another set of relations which determine the densities. These relations are most conveniently written in terms of the functions $\epsilon_{r}(\theta)$, which are defined by

$$
\frac{1}{1+e^{\epsilon_{r}}} \equiv \frac{\rho_{r}}{n_{r}}
$$

Notice that if the particles are free, $\rho_{r} / n_{r}$ is the Fermi distribution function. However, for $t \neq 2$, the particles are not free, and the $\epsilon_{r}$ are determined by the TBA equation

$$
\epsilon_{r}(\theta)=\frac{\mu_{r}}{\mu} e^{-\theta}-\frac{1}{2 \pi} \sum_{s} \int_{-\infty}^{\infty} d \theta^{\prime} \varphi_{r s}\left(\theta-\theta^{\prime}\right) \ln \left(1+e^{-\epsilon_{s}\left(\theta^{\prime}\right)}\right),
$$

where the label $s$ runs over breathers $(1 \ldots t-2)$ and \pm . The functions $\varphi_{r s}$ for integer $t$ are given in [31] or [22]. We will not need them, because here these equations can be written in a much simpler form [32]:

$$
\epsilon_{r}=\int_{-\infty}^{\infty} d \theta^{\prime} \frac{(t-1)}{2 \pi \cosh \left[(t-1)\left(\theta-\theta^{\prime}\right)\right]} \sum_{s} N_{r s} \ln \left(1+e^{\epsilon_{s}\left(\theta^{\prime}\right)}\right),
$$

where $N_{r s}$ is the incidence matrix of the following diagram

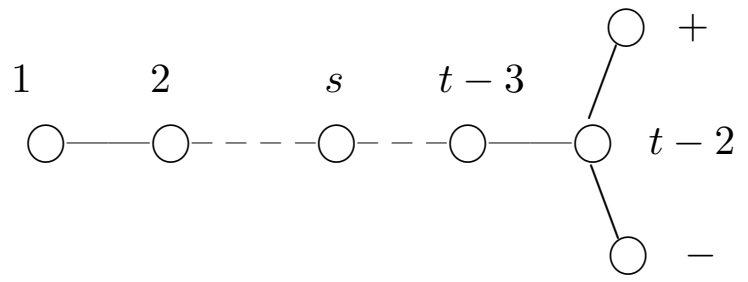

The dependences on the ratios $\mu_{a} / \mu$ seem to have disappeared from (4.2), but they appear as an asymptotic condition: the original equations (4.1) indicate that the solution must satisfy

$$
\epsilon_{r} \rightarrow \frac{\mu_{r}}{\mu} e^{-\theta} \quad \text { as } \theta \rightarrow-\infty
$$




\subsection{The partition function}

The impurity free energy is given in terms of $\epsilon_{+}$:

$$
\mathcal{F}_{T B A}=\frac{T_{B}}{2 \cos (\pi / 2(t-1))}-T \int \frac{d \theta}{2 \pi} \frac{t-1}{\cosh [(t-1)(\theta-\alpha)]} \ln \left(1+e^{\epsilon_{+}(\theta)}\right) .
$$

where $\alpha \equiv \ln \left(T / T_{B}\right)$ 1. The first piece is the non-analytic term (1.4). Since the same kernel appears in (4.4) and (4.2), $\mathcal{F}$ and $\mathcal{Z}$ can be written in a simpler form for many of the $t$. Using the relation (1.5) between $\mathcal{F}$ and $\mathcal{F}_{T B A}$, we have for example

$$
\begin{aligned}
& \mathcal{Z}(\alpha)=\sqrt{Y_{1}(\alpha) / 3} \quad t=3 \\
& \mathcal{Z}(\alpha)=\frac{1}{2}\left(Y_{2}(\alpha)\right)^{1 / 3} \quad t=4 \\
& \mathcal{Z}(\alpha)=\sqrt{Y_{3}(\alpha) / 5 Y_{1}(\alpha)} \quad t=5,
\end{aligned}
$$

where we define $Y_{r} \equiv \exp \left(\epsilon_{r}\right)$.

We derive simple functional relations for $\mathcal{Z}(\alpha)$ by continuing it and the $Y_{r}(\alpha)$ into the complex $\alpha$-plane [32]. Using the simple identity

$$
\lim _{x \rightarrow 0}\left[\frac{\lambda}{\cosh (\lambda \theta+i \pi / 2-x)}+\frac{\lambda}{\cosh (\lambda \theta-i \pi / 2+x)}\right]=2 \pi \delta(\theta)
$$

we have

$$
\mathcal{Z}(\alpha+\gamma) \mathcal{Z}(\alpha-\gamma)=\frac{1}{t}\left(1+Y_{+}(\alpha)\right)
$$

where $\gamma \equiv i \pi / 2(t-1)$. Similarly, the equations (4.2) yield

$$
\begin{aligned}
Y_{+}(\theta+\gamma) Y_{+}(\theta-\gamma) & =1+Y_{t-2}(\theta) \\
Y_{t-2}(\theta+\gamma) Y_{t-2}(\theta-\gamma) & =\left(1+Y_{t-3}(\theta)\right)\left(1+Y_{+}(\theta)\right)^{2} \\
Y_{a}(\theta+\gamma) Y_{a}(\theta-\gamma) & =\left(1+Y_{a+1}(\theta)\right)\left(1+Y_{a-1}(\theta)\right)
\end{aligned}
$$

where $a=1 \ldots t-3$, we define $Y_{0} \equiv 0$, and it follows from symmetry that $Y_{+}=Y_{-}$. These equations are applicable everywhere in the complex $\theta$-plane, whereas the original TBA equations apply only in a strip $|\operatorname{Im} \theta|<\pi /(t-1)$. The functional relations determine the functions $Y_{r}$ and $\mathcal{Z}$ uniquely once the asymptotic condition (4.3) is imposed. One can argue [32] that the functions $Y_{r}(\alpha)$ (and $\mathcal{Z}(\alpha)$ ) have the periodicity $Y_{r}(\alpha+t \gamma)=Y_{r}(\alpha)$,

1 This can be derived from the kernels $\kappa_{a}$ of [22] by using (4.2) along with the identity $2 \cosh y \tilde{\kappa}_{a}=\sum_{b} N_{a b} \tilde{\kappa}_{b}$ for $a=1 \ldots t-2$ and $2 \cosh y\left(\tilde{\kappa}_{+} \kappa_{-}\right)=2 \tilde{\kappa}_{t-2}+1$. 
which implies that they can be expanded in powers of $\left(T_{B} / T\right)^{-2(t-1) / t}$ for $T_{B} / T$ small. This then gives the expansions (2.1) and (2.13), because $x \propto\left(T_{B} / T\right)^{(t-1) / t}$.

Plugging the perturbative expansions into the functional relations (4.7) and (4.8) give non-trivial relations among the coefficients determined in sect. 2 by the Jack-polynomial expansions. For $t=3,4$ these constraints can be written in a simple form by using (4.5). Doing a little algebra, we have

$$
\begin{array}{lr}
3 \mathcal{Z}(\alpha+i \pi / 2) \mathcal{Z}(\alpha-i \pi / 2) \mathcal{Z}(\alpha)=\mathcal{Z}(\alpha+i \pi / 2)+\mathcal{Z}(\alpha)+\mathcal{Z}(\alpha-i \pi / 2) & t=3 \\
4 \mathcal{Z}(\alpha+i \pi / 3) \mathcal{Z}(\alpha-i \pi / 3) \mathcal{Z}(\alpha)=\mathcal{Z}(\alpha+i \pi / 3)+2 \mathcal{Z}^{2}(\alpha)+\mathcal{Z}(\alpha-i \pi / 3) & t=4 .
\end{array}
$$

These relations have the nice feature that they have lost all trace of the quasiparticle index $r$. This is a strong hint that they can be derived directly, without having to do the full TBA analysis. One can also hope that there is a simple relation even for non-integer $t$ (where the TBA analysis can get quite complicated); we make a conjecture in the next subsection. Even though (4.9) are stronger relations than (4.7) and (4.8), this still probably isn't the end of the story: the conjecture for $t=4$ amounts to $2 \mathcal{Z}(\alpha) \mathcal{Z}(\alpha+2 i \pi / 3)=$ $\mathcal{Z}(\alpha+i \pi / 3)+\mathcal{Z}(\alpha-i \pi / 3)$, which yields (4.9) but not the other way around.

Plugging (2.1) into (4.9), one finds

$$
\exp \left(-3 \sum_{n} f_{6 n} x^{6 n}\right)=\sum_{n} Z_{6 n} x^{6 n} \quad t=3 .
$$

This means, for example, that for $t=3,2 Z_{6}=-6 f_{6}=3 Z_{2} Z_{4}-\left(Z_{2}\right)^{3}$ and $Z_{12}=-3 f_{12}+$ $9\left(f_{6}\right)^{2} / 2$. Both agree with the Jack-polynomial expressions numerically evaluated in (2.14) and (2.15). In general, it means that that for $t=3$, the coefficients $Z_{6 n}$ are given in terms of the lower coefficients. Similarly, for $t=4$ one finds that the coefficients $Z_{4 n}$ are determined in terms of lower coefficients. For example, $Z_{4}=\left(Z_{2}\right)^{2} / 3=\pi /\left(3 \Gamma^{4}(3 / 4)\right)$ and $9 Z_{8}=18 Z_{2} Z_{6}-Z_{2}^{4}$. It would certainly be interesting to have a direct proof (i.e. one depending only on the expressions (2.7) or (2.8)) of these relations. They are certainly a hint of a much-deeper structure to the problem.

Since we cannot determine all of the coefficients from the functional relations, a final check is to solve the TBA equations numerically and then fit the results to a power series. Doing a perturbative expansion of the non-perturbative solution, one obtains

$$
\mathcal{F}_{T B A}=T \sum_{n=0}^{\infty} k_{2 n}\left(\frac{T_{B}}{T}\right)^{\left(1-\frac{1}{t}\right) 2 n}+\overline{\mathcal{F}} .
$$


We can now match these results with those of the Jack-polynomial expansion, once comparison of the first order has determined the ratio of the unknown constants $\kappa, \kappa^{\prime}$. We expect

$$
\frac{k_{2 n}}{f_{2 n}}=\left(\frac{k_{2}}{f_{2}}\right)^{n}
$$

We evaluate the full function $f(T)$ to double-precision accuracy by solving the integral equations (4.1), plugging this into the free energy (4.4), and then fitting this to the series (4.11) at large $T$. For $t=3$, we find

$$
\begin{array}{ll}
k_{2}=-.4567084 & k_{8}=0.000422 \\
k_{4}=0.0224220 & k_{10}=-0.00007 \\
k_{6}=-0.002818 &
\end{array}
$$

To the accuracy of the TBA fit, we have excellent agreement. The scales $x$ and $T_{B}$ are therefore related for $t=3$ by

$$
x^{2}=\left|k_{2}\right| \frac{\Gamma^{2}(2 / 3)}{\Gamma(1 / 3)}\left(\frac{T_{B}}{T}\right)^{4 / 3} .
$$

\subsection{The conductance}

In this subsection we use the non-perturbative TBA to derive a remarkable fluctuationdissipation relation of the conductance to the partition function. This allows us to obtain the value of infinitely-many coefficients in the perturbation expansion of $G$. It also allows us to conjecture a functional relation for $\mathcal{Z}$ for any rational value of $t$.

The TBA gives the conductance as [5]

$$
G(\alpha)=\int_{-\infty}^{\infty} d \theta \frac{t-1}{2 \cosh ^{2}[(t-1)(\theta-\alpha)]} \frac{1}{1+Y_{+}(\theta)}
$$

where $\alpha=\ln \left(T / T_{B}\right)$ as before, and $Y_{+}$is given by the TBA equations (4.2). By using the relation

$$
\lim _{x \rightarrow 0}\left[\frac{\lambda^{2}}{\cosh ^{2}(\lambda \theta+i \pi / 2-x)}-\frac{\lambda^{2}}{\cosh ^{2}(\lambda \theta-i \pi / 2+x)}\right]=-i 2 \pi \delta^{\prime}(\theta)
$$

one finds

$$
G(\alpha+\gamma)-G(\alpha-\gamma)=-i \frac{\pi}{t-1} \frac{\partial}{\partial \alpha} \frac{1}{1+Y_{+}(\alpha)}
$$


where $\gamma \equiv i \pi /(2(t-1))$. Using the relation (4.7), this gives

$$
G(\alpha+\gamma)-G(\alpha-\gamma)=-i \frac{\pi}{t(t-1)} \frac{\partial}{\partial \alpha}\left[\mathcal{Z}^{-1}(\alpha+\gamma) \mathcal{Z}^{-1}(\alpha-\gamma)\right]
$$

This fluctuation-dissipation relation has lost all trace of the quasiparticles of the TBA: it is thus tempting to conjecture that it holds for all $t$, not just the integer values where the TBA analysis is valid. The perturbative expansion is $G_{p e r t}=\sum_{n} g_{2 n} x^{2 n}$, so we have for example

$$
g_{2}=-Z_{2} \frac{2 \pi}{t^{2}} \cot \frac{\pi}{t}
$$

in agreement with the perturbative calculations (2.10) and (3.13), which are valid for any $t>2$.

When $t \equiv p / q$ is rational, this gives many but not all of the $g_{2 n}$ in terms of the $Z_{2 m}$ (with $m \leq n$ ), because the terms on the left-hand side of (4.15) vanish when $n$ is a multiple of $p$. For the physically-important value $t=3$, this gives all coefficients $g_{6 n+2}$ and $g_{6 n+4}$; for example

$$
g_{2}=-\frac{2 \pi}{9 \sqrt{3}} Z_{2} \quad g_{4}=\frac{4 \pi}{9 \sqrt{3}} Z_{4} \quad g_{8}=\frac{8 \pi}{9 \sqrt{3}}\left(Z_{8}-Z_{2} Z_{6}\right)
$$

These are in excellent agreement with a numerical calculation of the TBA conductance.

Although the fact that some of the terms on the left-hand side of (4.15) vanish for rational $t$ means we do not know how to relate these $g_{2 n}$ to the $Z_{2 n}$, it does seem to imply a constraint on $Z$. We know that $Z$ is an analytic function of $x$ for $t>2$, but we do not know that $G$ is as well. (One way of checking this would be to check that the explicit perturbative expansions (3.15) and (2.7) for $G$ and $\mathcal{Z}$ obey the formula (4.15).) If $G$ is indeed analytic (so $G=G_{\text {pert }}$ ), then it requires that these terms on the right-hand-side also vanish, which means that $\mathcal{Z}$ should satisfy

$$
\sum_{j=1}^{p} \mathcal{Z}^{-1}(\alpha+2 j \gamma) \mathcal{Z}^{-1}(\alpha+2(j-1) \gamma)=p
$$

For $t=3$ this is the relation already derived in (4.9), but for $t=4$ it is different. Putting it together with (4.9) for $t=4$, we find the simpler relation $2 \mathcal{Z}(\alpha) \mathcal{Z}(\alpha+2 i \pi / 3)=\mathcal{Z}(\alpha+$ $i \pi / 3)+\mathcal{Z}(\alpha-i \pi / 3)$. This relation alone implies both (4.16) and (4.9) for $t=4$. We can check the relationship (4.16) numerically. Plugging the expansion of $\mathcal{Z}$ into (4.16) gives the coefficients $Z_{2 p}$ in terms of lower ones, which can be compared with the expression 
(2.7). We have checked $Z_{10}$ for $t=5$ and $t=5 / 2$, and find that it is indeed satisfied. This leads us to conjecture that (4.16) is true for all $t>2$ and rational. We also note that the relations (4.7) and (4.8) require that $\mathcal{Z}$ should obey an even more restrictive functional relationship. We have not succeeded in finding its general form, but one can always plug the perturbative expansions into (4.7) and (4.8) to derive more relations among the coefficients.

To conclude this section, we recall first that the TBA analysis is usually made for $t$ rational only. Moreover some of the results given above hold for $t$ integer only. However some of the functional relations we have uncovered seem to make sense for any $t$. Observe also that the Jack expansion and TBA behave differently as $t \rightarrow 2$. In the former case all integrals just blow up, while in the latter one gets finite results for the free energy, involving however logarithmic terms. Presumably, the TBA gives the regularized version of the Jack computations.

\section{Conclusion}

Using Jack polynomials and the thermodynamic Bethe ansatz, many properties of the 1d log-sine gas can be computed exactly, some of which are of experimental significance. We hope that these methods can be used for other problems with potential applications, in particular for dissipative quantum mechanics. Moreover, multiple integrals similar to those we do using the Jack symmetric functions appear in many different kinds of computations, so we hope that these techniques are generally applicable.

On the more formal side, it is exciting to have an example where two different areas of mathematical physics meet. By analogy, one might hope that these TBA techniques can be applied to other $1 / r^{2}$ models, like the Calogero-Sutherland model, where Jack polynomials have been used recently. This overlap of techniques has led to intriguing relations between various quantities of Jack symmetric function theory. For example, when $t=4$ the series (2.7) can be summed to give $Z_{4}=\pi /\left(3 \Gamma^{4}(3 / 4)\right)$, but we have no direct proof, so we do not know if this is a fluke or if a closed-form expression can be found for all $t$. The overlap has also led to several simple but powerful conjectures like (4.16) and (3.14). One can hope that this is evidence of a more complete mathematical structure behind the scene.

Acknowledgments: H.S. is grateful to Saclay for its warm hospitality when this work was started, and to I. Kostov and V. Pasquier for very useful discussions. F.L. thanks P. Di Francesco for discussions. This work was supported by the Packard Foundation, the National Young Investigator program (NSF-PHY-9357207) the DOE (DE-FG03-84ER40168), and a Canadian NSERC 67 scholarship (F.L.). 


\section{References}

[1] M.L. Mehta, Random matrices, Academic Press (NY) 1991.

[2] P.W. Anderson, G. Yuval and D.R. Hamman, Phys. Rev. B1 (1970) 4464.

[3] C.L. Kane and M.P.A. Fisher, Phys. Rev. B46 (1992) 15233.

[4] K. Moon, H. Yi, C.L. Kane, S.M. Girvin and M.P.A. Fisher, Phys. Rev. Lett. 71 (1993) 4381.

[5] P. Fendley, A. Ludwig and H. Saleur, "Exact Conductance through Point Contacts in the $\nu=1 / 3$ Fractional Quantum Hall Effect", USC-94-12, PUPT-94-1491, condmat/9408068.

[6] A. Schmid, Phys. Rev. Lett. 51 (1983) 1506.

[7] M.P.A. Fisher and W. Zwerger, Phys. Rev. B 32 (1985) 6190.

[8] F. Guinea, V. Hakim and A. Muramatsu, Phys. Rev. Lett. 54 (1985) 263.

[9] F. Guinea, Phys. Rev. B32 (1985) 7518.

[10] C. Callan and D. Freed, Nucl. Phys. B374 (1992) 543.

[11] N. Andrei, K. Furuya, and J. Lowenstein, Rev. Mod. Phys. 55 (1983) 331;

A.M. Tsvelick and P.B. Wiegmann, Adv. Phys. 32 (1983) 453.

[12] I.G. Macdonald, Seminaire Lotharingien, Publ. I.R.M.A., Strasbourg 1988.

[13] I.G. Macdonald, Symmetric functions and Hall polynomials, Clarendon Press (1979).

[14] R.P. Stanley, Adv. in Math. 77 (1989) 76.

[15] K.W.J. Kadell, Compos. Math. 87 (1993) 5.

[16] F. Calogero, J. Math. Phys. 10 (1969) 2191.

[17] B. Sutherland, J. Math. Phys. 12 (1977) 246, 251.

[18] F.D.M. Haldane, Phys. Rev. Lett. 66 (1991) 1529.

[19] P.J. Forrester, Nucl. Phys. B388 (1992) 671.

[20] F. Lesage, V. Pasquier and D. Serban, "Dynamical correlation functions in the Calogero-Sutherland model", hep-th/9405008.

[21] Z.N.C. Ha, Phys. Rev. Lett. 73 (1994) 1574, cond-mat/9405063.

[22] P. Fendley, H. Saleur and N.P. Warner, "Exact solution of a massless scalar field with a relevant boundary interaction", to appear in Nucl. Phys. B, hep-th/9406125.

[23] C.N. Yang and C.P. Yang, J. Math. Phys. 10 (1969) 1115.

[24] Al.B. Zamolodchikov, Nucl. Phys. B342 (1991) 695.

[25] I. Affleck and A. Ludwig, Phys. Rev. Lett. 67 (1991) 161.

[26] E. Wong and I. Affleck, Nucl. Phys. B417 (1994) 403, cond-mat/9311040.

[27] J.L. Cardy, J. Phys. A14 (1981) 1407.

[28] K.H. Kjaer and H.J.Hilhorst, J. Stat. Phys. 28 (1982) 621.

[29] C. Itzykson and H. Saleur, J.Stat. Phys. 48 (1987) 449.

[30] S. Ghoshal and A.B. Zamolodchikov, Int. J. Mod. Phys. A9 (1994) 3841, hepth/9306002.

[31] T. Klassen and E. Melzer, Nucl. Phys. B338 (1990) 485.

[32] Al.B. Zamolodchikov, Phys. Lett. B253 (1991) 391. 